\title{
Time-dependent HF approach to SHE dynamics
}

\author{
A.S. Umar ${ }^{\mathrm{a}}$, V.E. Oberacker ${ }^{\mathrm{a}}$ \\ ${ }^{a}$ Department of Physics and Astronomy, Vanderbilt University, Nashville, TN 37235, USA
}

\begin{abstract}
We employ the time-dependent Hartree-Fock (TDHF) method to study various aspects of the reactions utilized in searches for superheavy elements. These include capture cross-sections, quasifission, prediction of $P_{\mathrm{CN}}$, and other interesting dynamical quantities. We show that the microscopic TDHF approach provides an important tool to shed some light on the nuclear dynamics leading to the formation of superheavy elements.
\end{abstract}

Keywords: TDHF; TDDFT; SHE; Superheavy nuclei, Quasifission

\section{Introduction}

The search for new elements is one of the most novel and challenging research areas of nuclear physics [1-4]. The discovery of a region of the nuclear chart that can sustain the so called superheavy elements (SHE) has lead to intense experimental activity resulting in the discovery of elements with atomic numbers as large as $Z=117$ [5-7]. The theoretically predicted island of stability is the result of new proton and neutron shell-closures, whose location is not precisely known [8-13]. The experiments to discover these new elements are notoriously difficult, with production cross-sections in pico-barns. Of primary importance for the experimental investigations appear to be the choice of target-projectile combinations that have the highest probability for forming a compound nucleus that results in the production of the desired element. Experimentally, two approaches have been used for the synthesis of these elements, one utilizing doubly-magic ${ }^{208} \mathrm{~Pb}$ targets or ${ }^{209} \mathrm{Bi}$ (cold-fusion) [3, 14, 15], the other utilizing deformed actinide targets with neutron-rich projectiles (hot-fusion), such as ${ }^{48} \mathrm{Ca}$ [4, 16, 17]. While both methods have been successful in synthesizing new elements the evaporation residue crosssections for hot-fusion were found to be several orders of magnitude larger than those for cold fusion. To pinpoint the root of this difference it is important to understand the details of the reaction dynamics of these systems. For light and medium mass systems the capture cross-section may be considered to be the same as that for complete fusion, whereas for heavy systems leading to superheavy formations the evaporation residue cross-section is dramatically reduced due to the quasifission $(\mathrm{QF})$ and fusion-fission processes [18-24] thus making the capture cross-section to be essentially the sum of these two cross-sections, with QF occurring at a much shorter timescale.. Consequently, quasifission is the primary reaction mechanism that limits the formation of superheavy nuclei. Various theoretical models have been developed to study the quasifission process [25-31].

Email address: umar@compsci.cas.vanderbilt.edu (A.S. Umar)

Preprint submitted to Nuclear Physics A

February 12, 2015

(C) 2015. This manuscript version is made available under the Elsevier user license

http://www.elsevier.com/open-access/userlicense/1.0/ 
In many branches of science, highly complex many-body systems are often described in macroscopic terms, which is particularly true in the case of non-relativistic heavy-ion collisions. For example, the time evolution of the collective nuclear surface variables $\alpha_{\ell m}(t)$ and the corresponding geometrical nuclear shape $R(\theta, \phi, t)$ provides a very useful set of parameters to help organize experimental data. Using this approach numerous evolutionary models have been developed to explain particular aspects of experimental data. These methods provide a useful and productive means for quantifying multitudinous reaction data. In practice, they require a quantitative understanding of the data as well as a clear physical picture of the important aspects of the reaction dynamics. The depiction of the collision must be given at the onset, including the choice of coordinates which govern the evolution of the reaction. Guessing the correct degrees of freedom is extremely hard, without a full understanding of the dynamics, and can easily lead to misbegotten results. More importantly, it is most often not possible to connect these macroscopic classical parameters, describing nuclear matter under extreme excitation and rearrangement, with the more fundamental properties of the nuclear force. Such difficulties can only be overcome with a fully microscopic theory of the collision dynamics.

The theoretical formalism for the microscopic description of complex many-body quantum systems and the understanding of the nuclear interactions that result in self-bound, composite nuclei possessing the observed properties are the underlying challenges for studying low energy nuclear physics. The Hartree-Fock approximation and its time-dependent generalization, the time-dependent Hartree-Fock theory, has provided a possible means to study the diverse phenomena observed in low energy nuclear physics [32, 33]. In general TDHF theory provides a useful foundation for a fully microscopic many-body theory of large amplitude collective motion including collective surface vibrations and giant resonances [34-42], nuclear reactions in the vicinity of the Coulomb barrier, such as fusion [43-48], deep-inelastic reactions and transfer [49. 53], and dynamics of fission fragments [54]. As a result of theoretical approximations (single Slater determinant), TDHF does not describe individual reaction channels; rather, it describes the time-evolution of the dominant reaction channel. In other words TDHF is a deterministic theory. To obtain multiple reaction channels or widths of observables one must go beyond TDHF [50, 55-57]. In connection with superheavy element formation, the theory predicts best the cross-section for a particular process which dominates the reaction mechanism. This is certainly the case for studying capture cross-sections and quasifission.

In recent years has it become numerically feasible to perform TDHF calculations on a 3D Cartesian grid without any symmetry restrictions and with much more accurate numerical methods [33, 58, 60]. In addition, the quality of effective interactions has been substantially improved [61-63]. While ordinary TDHF calculations can be used for fusion or capture at energies above the barrier they cannot be used directly at sub-barrier energies. During the past several years, a novel approach based on TDHF called the density constrained time-dependent HartreeFock (DC-TDHF) method was developed to compute heavy-ion potentials [64] and excitation energies [65] directly from TDHF time-evolution. This method was applied to calculate fusion and capture cross sections above and below the barrier, ranging from light and medium mass systems [45, 66-70] to hot and cold fusion reactions leading to superheavy element $Z=112$ [43]. In all cases a good agreement between the measured fusion cross sections and the DC-TDHF results was found. This is rather remarkable given the fact that the only input in DC-TDHF is the Skyrme effective N-N interaction, whose parameters are determined from static structure information and there are no adjustable parameters.

Within the last few years the TDHF approach has been utilized for studying the dynamics of quasifission [71, 72] and scission [54]. Particularly, the study of quasifission is showing a great 
promise to provide insight based on very favorable comparisons with experimental data. In this article we will focus on the TDHF studies of capture cross-sections, quasifission observables, and related quantities.

\section{Theory}

We now give a brief outline of the TDHF method and some of the recent extensions used in the calculations presented [32, 33]. Given a many-body Hamiltonian $H$ containing two and three-body interactions the time-dependent action $S$ can be constructed as

$$
S=\int_{t_{1}}^{t_{2}} d t<\Phi(t)\left|H-i \hbar \partial_{t}\right| \Phi(t)>.
$$

Here, $\Phi$ denotes the time-dependent many-body wavefunction $\Phi\left(\mathbf{r}_{1}, \mathbf{r}_{2}, \ldots, \mathbf{r}_{\mathrm{A}} ; t\right)$. General variation of $S$ recovers the time-dependent Schrödinger equation. In TDHF approximation the manybody wavefunction is replaced by a single Slater determinant and this form is preserved at all times, implying that two-body correlations are neglected. The determinental form guarantees the antisymmetry required by the Pauli principle for a system of fermions. In this limit, the variation of the action yields the most probable time-dependent path between points $t_{1}$ and $t_{2}$ in the multi-dimensional space-time phase space

$$
\delta S=0 \rightarrow \Phi(t)=\Phi_{0}(t) .
$$

In practice $\Phi_{0}(t)$ is chosen to be a Slater determinant comprised of single-particle states $\phi_{\lambda}(\mathbf{r}, t)$ with quantum numbers $\lambda$. If the variation in Eq. (2) is performed with respect to the singleparticle states $\phi_{\lambda}^{*}$ we obtain a set of coupled, nonlinear, self-consistent initial value equations for the single-particle states

$$
h\left(\left\{\phi_{\mu}\right\}\right) \phi_{\lambda}=i \hbar \dot{\phi_{\lambda}} \quad \lambda=1, \ldots, N \text {. }
$$

These are the fully microscopic time-dependent Hartree-Fock equations which preserve the major conservation laws such as the particle number, total energy, total angular momentum, etc. As we see from Eq.(3), each single-particle state evolves in the mean-field $h$ generated by the concerted action of all the other single-particle states.

In TDHF, the initial nuclei are calculated by solving the static Hartree-Fock (HF) equations using the damped-relaxation method [73, 74]. The resulting Slater determinants for each nucleus comprise the larger Slater determinant describing the colliding system during the TDHF evolution. Nuclei are assumed to move on a pure Coulomb trajectory until the initial separation between the nuclear centers used in TDHF evolution. Using the Coulomb trajectory we compute the relative kinetic energy at this separation and the associated translational momenta for each nucleus. The nuclei are then boosted by multiplying the HF states with a phase factor

$$
\Phi_{j} \rightarrow \exp \left(l \mathbf{k}_{j} \cdot \mathbf{R}\right) \Phi_{j}
$$

where $\Phi_{j}$ is the HF state for nucleus $j$ and $\mathbf{R}$ is the corresponding center of mass coordinate

$$
\mathbf{R}=\frac{1}{A_{j}} \sum_{i=1}^{A_{j}} \mathbf{r}_{i}
$$

The Galilean invariance of the TDHF equations assures the evolution of the system without spreading and the conservation of the total energy for the system. In TDHF, the many-body state remains a Slater determinant at all times. 


\subsection{DC-TDHF method}

The concept of using density as a constraint for calculating collective states from TDHF time-evolution was first introduced in [75], and used in calculating collective energy surfaces in connection with nuclear molecular resonances in [76].

In this approach we assume that a collective state is characterized only by density $\rho$ and current $\mathbf{j}$. This state can be constructed by solving the static Hartree-Fock equations

$$
<\Phi_{\rho, \mathbf{j}}\left|a_{h}^{\dagger} a_{p} \hat{H}\right| \Phi_{\rho, \mathbf{j}}>=0,
$$

subject to constraints on density and current

$$
\begin{aligned}
& <\Phi_{\rho, \mathbf{j}}|\hat{\rho}(\mathbf{r})| \Phi_{\rho, \mathbf{j}}>=\rho(\mathbf{r}, t) \\
& <\Phi_{\rho, \mathbf{j}}|\hat{\jmath}(\mathbf{r})| \Phi_{\rho, \mathbf{j}}>=\mathbf{j}(\mathbf{r}, t) .
\end{aligned}
$$

Choosing $\rho(\mathbf{r}, t)$ and $\mathbf{j}(\mathbf{r}, t)$ to be the instantaneous TDHF density and current results in the lowest energy collective state corresponding to the instantaneous TDHF state $|\Phi(t)\rangle$, with the corresponding energy

$$
E_{\text {coll }}(\rho(t), \mathbf{j}(t))=<\Phi_{\rho, \mathbf{j}}|\hat{H}| \Phi_{\rho, \mathbf{j}}>.
$$

This collective energy differs from the conserved TDHF energy only by the amount of internal excitation present in the TDHF state, namely

$$
E^{*}(t)=E_{T D H F}-E_{\text {coll }}(t) .
$$

However, in practical calculations the constraint on the current is difficult to implement but we can define instead a static adiabatic collective state $\mid \Phi_{\rho}>$ subject to the constraints

$$
\begin{aligned}
& <\Phi_{\rho}|\hat{\rho}(\mathbf{r})| \Phi_{\rho}>=\rho(\mathbf{r}, t) \\
& <\Phi_{\rho}|\hat{\jmath}(\mathbf{r})| \Phi_{\rho}>=0 .
\end{aligned}
$$

In terms of this state one can write the collective energy as

$$
E_{\text {coll }}(t)=E_{\text {kin }}(\rho(t), \mathbf{j}(t))+E_{D C}(\rho(\mathbf{r}, t)),
$$

where the density-constrained energy $E_{D C}$, and the collective kinetic energy $E_{k i n}$ are defined as

$$
\begin{aligned}
E_{D C} & =<\Phi_{\rho}|\hat{H}| \Phi_{\rho}> \\
E_{k i n}(t) & \approx \frac{m}{2} \sum_{q} \int d^{3} r \mathbf{j}_{q}^{2}(t) / \rho_{q}(t)
\end{aligned}
$$

where the index $q$ is the isospin index for neutrons and protons $(q=n, p)$. From Eq. 9 it is clear that the density-constrained energy $E_{D C}$ plays the role of a collective potential. In fact this is exactly the case except for the fact that it contains the binding energies of the two colliding nuclei. One can thus define the ion-ion potential as [64]

$$
V(R(t))=E_{\mathrm{DC}}(\rho(\mathbf{r}, t))-E_{A_{1}}-E_{A_{2}},
$$

where $E_{A_{1}}$ and $E_{A_{2}}$ are the binding energies of two nuclei obtained from a static Hartree-Fock calculation with the same effective interaction. For describing a collision of two nuclei one can 
label the above potential with ion-ion separation distance $R(t)$ obtained during the TDHF timeevolution. This ion-ion potential $V(R)$ is asymptotically correct since at large initial separations it exactly reproduces $V_{\text {Coulomb }}\left(R_{\text {max }}\right)$. In addition to the ion-ion potential it is also possible to obtain coordinate dependent mass parameters. One can compute the "effective mass" $M(R)$ using the conservation of energy in a central collision

$$
M(R)=\frac{2\left[E_{\mathrm{c} . \mathrm{m} .}-V(R)\right]}{\dot{R}^{2}},
$$

where the collective velocity $\dot{R}$ is directly obtained from the TDHF time evolution and the potential $V(R)$ from the density constraint calculations. Recently, a new DC-TDHF study was done to include the non-central effects in to the calculation of $M(R)$ [77], which found improvements at above barrier energies.

In practice, the potential barrier penetrabilities $T_{L}$ at $E_{\mathrm{c} . \mathrm{m} \text {. }}$ energies below and above the barrier are obtained by numerical integration of the Schrödinger equation for the relative coordinate $R$ using the well-established Incoming Wave Boundary Condition (IWBC) method [78].

\subsection{Skyrme interaction}

Almost all TDHF calculations have been done using the Skyrme energy density functional. The Skyrme energy density functional contains terms which depend on the nuclear density, $\rho$, kinetic-energy density, $\tau$, spin density, $\mathbf{s}$, spin kinetic energy density, $\mathbf{T}$, and the full spin-current pseudotensor, J, as

$$
E=\int d^{3} r \mathcal{H}(\rho, \tau, \mathbf{j}, \mathbf{s}, \mathbf{T}, \mathbf{J} ; \mathbf{r})
$$

The time-odd terms $(\mathbf{j}, \mathbf{s}, \mathbf{T})$ vanish for static calculations of even-even nuclei, while they are present for odd mass nuclei, in cranking calculations, as well as in TDHF. The spin-current pseudotensor, $\mathbf{J}$, is time-even and does not vanish for static calculations of even-even nuclei. It has been shown [40,59,79, 82] that the presence of these extra terms are necessary for preserving the Galilean invariance and make an appreciable contribution to the dissipative properties of the collision. Our TDHF program includes all of the appropriate combinations of time-odd terms in the Skyrme interaction. In addition, commonly a pairing force is added to incorporate pairing interactions for nuclei. The implementation of pairing for time-dependent collisions is currently an unresolved problem although small amplitude implementations exist [83-85] The implementation of pairing for collisions has been recently studied at the TDHF+BCS level by Scamps and Lacroix [53]. However, for reactions with relatively high excitation this is not expected to be a problem.

\section{Capture cross-sections}

For the reactions of heavy systems the process of overcoming the interaction barrier is commonly referred to as capture. After capture a number of possibilities exist [86]

$$
\begin{aligned}
\sigma_{\text {capture }} & =\sigma_{\text {fusion }}+\sigma_{\text {quasifission }}+\sigma_{\text {fastfission }} \\
\sigma_{\text {fusion }} & =\sigma_{\text {evaporation residue }}+\sigma_{\text {fusion-fission }}
\end{aligned}
$$

Understanding each of these cross-sections is vital for selecting reaction partners that have the highest probability for producing a superheavy element. As we have discussed above one of 
the applications of the DC-TDHF method is the calculation of microscopic potential barriers for reactions leading to superheavy formations. This allows the calculation of capture crosssections and the excitation energy of the system at the capture point. In this section we briefly describe capture cross-section calculations and in the following sections we will show results for quasifission and the possibility of calculating some of the ingredients for the calculation of $P_{\mathrm{CN}}$.

In connection with superheavy element production, we have studied the hot fusion reaction ${ }^{48} \mathrm{Ca}+{ }^{238} \mathrm{U}$ and the cold fusion reaction ${ }^{70} \mathrm{Zn}+{ }^{208} \mathrm{~Pb}[43]$. Considering hot fusion, ${ }^{48} \mathrm{Ca}$ is a spherical nucleus whereas ${ }^{238} \mathrm{U}$ has a large axial deformation. The deformation of ${ }^{238} \mathrm{U}$ strongly influences the interaction barrier for this system. This is shown in the left panel of Fig. 1. which shows the interaction barriers, $V(R)$, calculated using the DC-TDHF method as a function of c.m. energy and for three different orientations of the ${ }^{238} \mathrm{U}$ nucleus. The alignment angle $\beta$ is the angle between the symmetry axis of the ${ }^{238} \mathrm{U}$ nucleus and the collision axis. Also, shown in the left panel of Fig. 1] are the experimental energies [4, 17] for this reaction. We observe that all of the experimental energies are above the barriers obtained for the polar alignment of the ${ }^{238} \mathrm{U}$ nucleus.
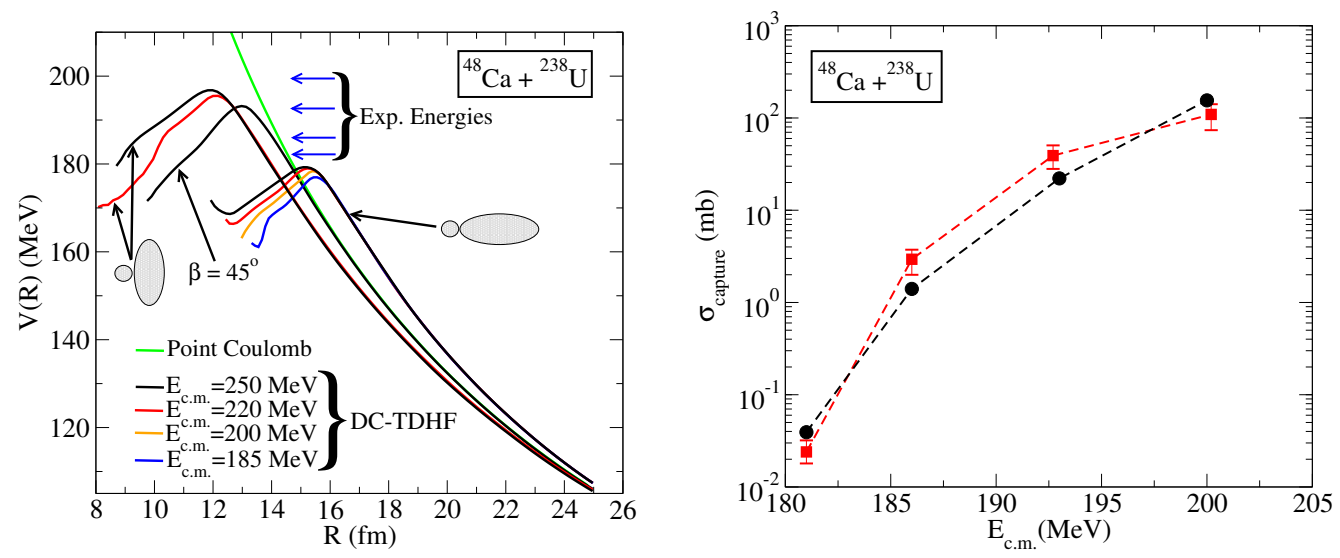

Figure 1: Left: Potential barriers, $V(R)$, obtained from DC-TDHF calculations [43] as a function of $E_{\text {c.m. }}$ energy and orientation angle $\beta$ of the ${ }^{238} \mathrm{U}$ nucleus. Also shown are the experimental c.m. energies. Right: Capture cross-sections as a function of $E_{\text {c.m. }}$ energy (black circles). Also shown are the experimental cross-sections [4] 87, 88] (red squares).

The barriers for the polar orientation $\left(\beta=0^{\circ}\right)$ of the ${ }^{238} \mathrm{U}$ nucleus are much lower and peak at larger ion-ion separation distance $R$. On the other hand, the barriers for the equatorial orientation $\left(\beta=90^{\circ}\right)$ are higher and peak at smaller $R$ values. We observe that at lower energies the polar orientation results in sticking of the two nuclei, while the equatorial orientation results in a deepinelastic collision. We have also calculated the excitation energy $E^{*}(R)$ as a function of c.m. energy and orientation angle $\beta$ of the ${ }^{238} \mathrm{U}$ nucleus. The system is excited much earlier during the collision process for the polar orientation and has a higher excitation than the corresponding collision for the equatorial orientation.

To obtain the capture cross-section, we calculate potential barriers $V(R, \beta)$ for a set of initial orientations $\beta$ of the ${ }^{238} \mathrm{U}$ nucleus. Then we determine partial cross sections $\sigma(\beta)$ and perform an angle-average. However, as a result of long-range Coulomb excitation, not all initial orientation angles occur with the same probability. Rather, the dominant excitation of the ground state rotational band in deformed nuclei leads to a preferential alignment which is calculated in a 
separate semiclassical Coulomb excitation code [66]. This code is only used to determine the alignment probability $\mathrm{d} P /(\mathrm{d} \beta \sin \beta)$ in Eq. 13 for the angle averaging of the cross-section

$$
\sigma_{\text {capture }}\left(E_{\text {c.m. }}\right)=\int_{0}^{\pi} \mathrm{d} \beta \sin \beta \frac{\mathrm{d} P}{\mathrm{~d} \beta \sin \beta} \sigma\left(E_{\mathrm{c} . \mathrm{m} .}, \beta\right)
$$

and $\sigma\left(E_{\mathrm{c} . \mathrm{m} .}, \beta\right)$ is the capture cross-section associated with a particular alignment. For heavy systems the alignment probability due to rotational excitations can be substantially different than an isotropic distribution [43, 89] In the right panel of Fig. 1] we show our results for the capture cross-sections which are in remarkably good agreement with experimental data.

\section{Quasifission}

The feasibility of using TDHF for quasifission has only been recognized recently [33, 71, 72]. By virtue of long contact-times for quasifission and the energy and impact parameter dependence these calculations require extremely long CPU times and numerical accuracy [58, 60, 73, 80, 81].

\section{1. $C a+U$ systems}

In the present TDHF calculations we use the Skyrme SLy4d interaction [90] including all of the relevant time-odd terms in the mean-field Hamiltonian. First we generate very accurate static $\mathrm{HF}$ wave functions for the two nuclei on the 3D grid. The initial separation of the two nuclei

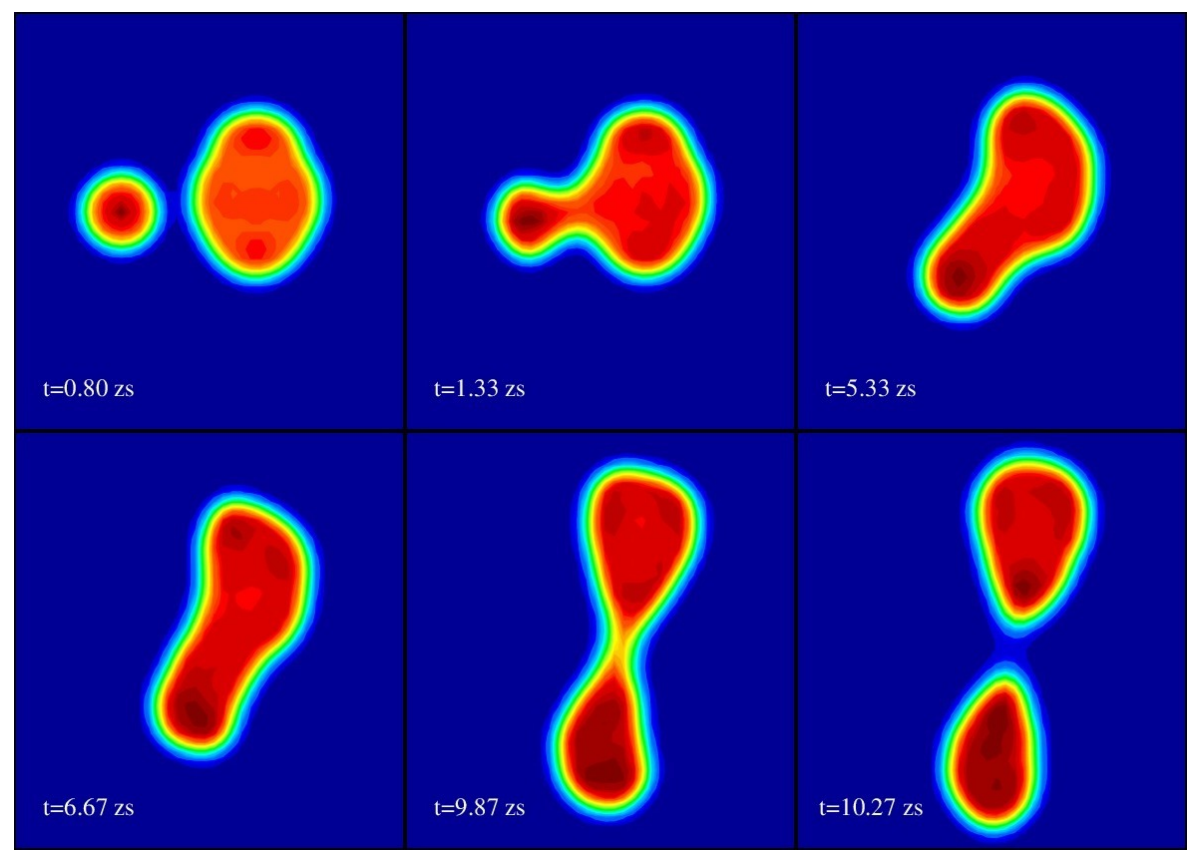

Figure 2: Quasifission in the reaction ${ }^{40} \mathrm{Ca}+{ }^{238} \mathrm{U}$ at $E_{\text {c.m. }}=209 \mathrm{MeV}$ with impact parameter $b=1.103 \mathrm{fm}(L=20)$ Shown is a contour plot of the time evolution of the mass density. Time increases from left to right and top to bottom. The actual numerical box is larger than the ones shown in the figure.

is $30 \mathrm{fm}$. In the second step, we apply a boost operator to the single-particle wave functions. 
The time-propagation is carried out using a Taylor series expansion (up to orders $10-12$ ) of the unitary mean-field propagator, with a time step $\Delta t=0.4 \mathrm{fm} / \mathrm{c}$. In Fig. 2 we show contour plots of the mass density for the ${ }^{40} \mathrm{Ca}+{ }^{238} \mathrm{U}$ reaction at $E_{\text {c.m. }}=209 \mathrm{MeV}$ as a function of time. The impact parameter $b=1.103 \mathrm{fm}$ corresponds to an average orbital angular momentum quantum number $L=20$. In this case, the $3 \mathrm{D}$ lattice spans $(66 \times 56 \times 30) \mathrm{fm}$. As the nuclei approach each other, a neck forms between the two fragments which grows in size as the system begins to rotate. Due to the centrifugal forces the dinuclear system elongates and forms a very long neck which eventually ruptures leading to two separated fragments. The ${ }^{238} \mathrm{U}$ nucleus exhibits both quadrupole and hexadecapole deformation; in the present study, its symmetry axis was oriented initially at $90^{\circ}$ to the internuclear axis. This orientation leads to the largest "contact time" [33] which is defined as the time interval between the time $t_{1}$ when the two nuclear surfaces first touch and the time $t_{2}$ when the dinuclear system splits up again. In this case, we find a contact time $\Delta t=9.35 \mathrm{zs}$ ( $1 \mathrm{zs}$ $=10^{-21} \mathrm{~s}$ ) and substantial mass transfer ( 66 nucleons to the light fragment). The event has all the characteristics of quasifission. The orientation of the ${ }^{238} \mathrm{U}$ symmetry axis at $0^{\circ}$ to the internuclear axis also results in QF but with much shorter contact-times and consequently with smaller mass transfer. These contribute more to the large asymmetric part of the mass distribution [71] and will not be considered for the purposes of this study.

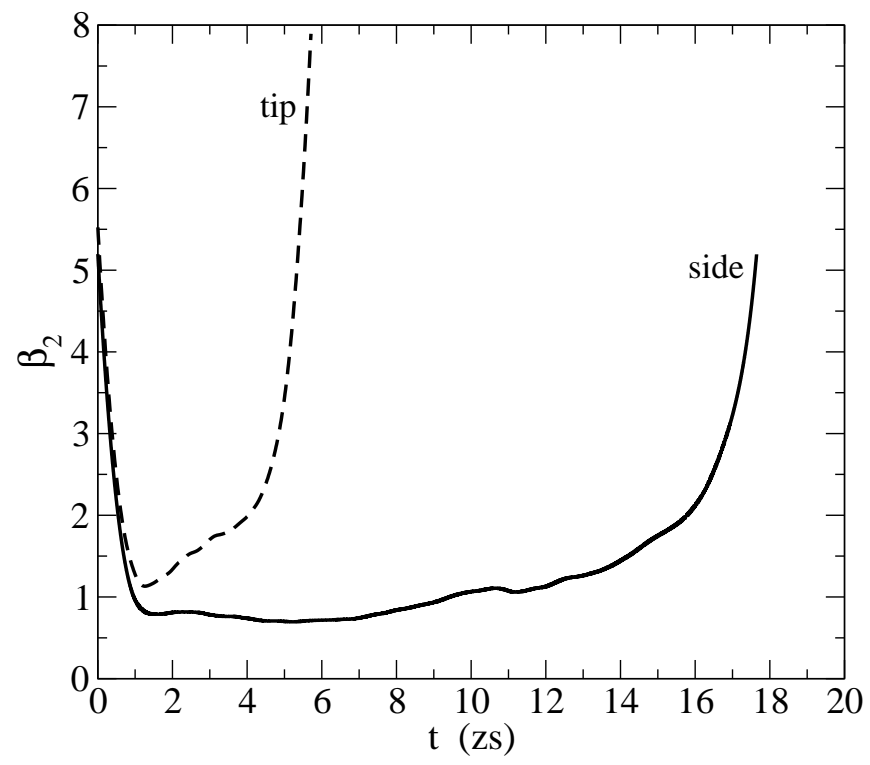

Figure 3: TDHF results showing the time-dependence of the deformation $\beta_{2}$ for a central collision of ${ }^{48} \mathrm{Ca}+{ }^{238} \mathrm{U}$ at $E_{\text {c.m. }}=203 \mathrm{MeV}$. The two curves show the two orientations of the ${ }^{238} \mathrm{U}$ nucleus with respect to the collision axis.

Another interesting observable is the time-evolution of the quasifissioning system. In order to have the correct quadrupole moment for a changing nuclear density one has to diagonalize the mass quadrupole tensor matrix

$$
Q_{i j}=\int d^{3} r \rho_{T D H F}(\mathbf{r}, t)\left(3 x_{i} x_{j}-r^{2} \delta_{i j}\right)
$$

The largest eigenvalue gives the mass quadrupole moment, $Q_{20}$, calculated along the principal 
axis for the nucleus (after multiplying with $\sqrt{5 / 16 \pi}$ ). From these one can construct the deformation parameter $\beta_{2}$

$$
\beta_{2}=\frac{4 \pi}{3} \frac{Q_{20}}{A R_{0}^{2}},
$$

where $R_{0}=1.2 A^{1 / 3}$. The changing quadrupole moment during the collision, particularly towards the last stages of the quasifission process shows not only the elongation of the nucleus but the rate of change also shows the velocity by which the quasifission event is taking place. In Fig. 3 we plot the TDHF results showing the time-dependence of the deformation $\beta_{2}$ for a central collision of ${ }^{48} \mathrm{Ca}+{ }^{238} \mathrm{U}$ at $E_{\text {c.m. }}=203 \mathrm{MeV}$. The two curves show the two orientations of the ${ }^{238} \mathrm{U}$ nucleus with respect to the collision axis. One clearly observes that the neutron-rich system stays at a compact shape much longer than the neutron-poor system and the actual quasifission event happens relatively quickly.

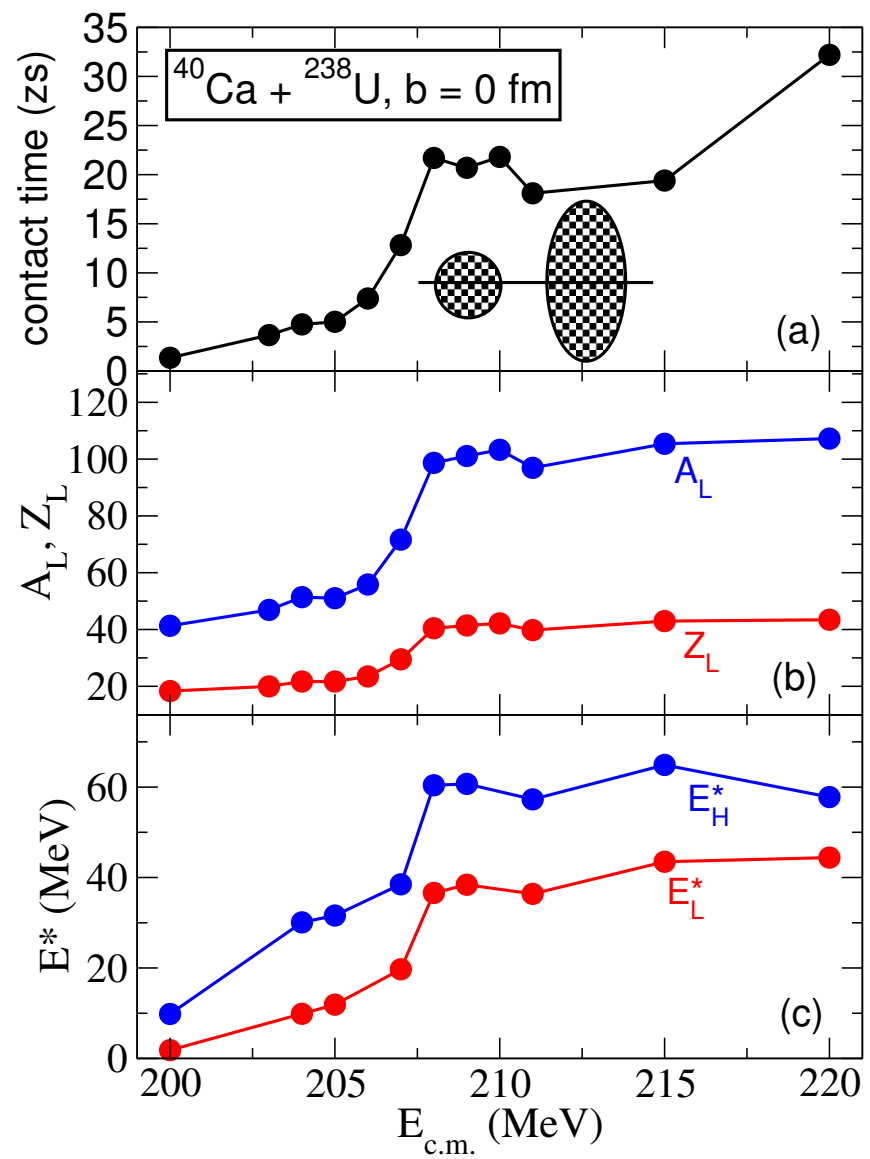

Figure 4: Several observables as a function of center-of-mass energy for a central collision of ${ }^{40} \mathrm{Ca}+{ }^{238} \mathrm{U}[\mathbf{7 2 ]}$. (a) contact time, (b) mass and charge of the light fragment, and (c) excitation energy of the light and heavy fragments. Quasifission dominates in the energy region $E_{\mathrm{c} . \mathrm{m} .}=208-220 \mathrm{MeV}$.

Recently, we have studied the central and non-central collisions of ${ }^{40} \mathrm{Ca}+{ }^{238} \mathrm{U}$ [72]. In the energy interval $E_{\mathrm{c} . \mathrm{m} .}=200-220 \mathrm{MeV}$ we always observe two fragments in the exit channel, i.e. 
these events are either a deep-inelastic reaction or quasifission. The distinction between deepinelastic or quasifission can be characterized by a number of factors; experimentally a large mass transfer (e.g. $A>20$ ) can be used to distinguish the two. In addition mass-angle distribution plots can be used to identify fragments undergoing large rotations. Finally, the TKE distribution of the final fragments and their agreement with the Viola distribution (see below) is a strong indicator. In Fig. 4 a we display the contact time as a function of the center-of-mass energy. We observe that in the energy interval $E_{\text {c.m. }}=200-205 \mathrm{MeV}$ the contact time increases slowly with increasing energy. Between $E_{\mathrm{c} . \mathrm{m} .}=205-208 \mathrm{MeV}$ there is a steep increase in the contact time. In the energy range $E_{\mathrm{c} . \mathrm{m} .}=208-220 \mathrm{MeV}$ the contact time varies between (21.7-32.2) zs which is (16-24) times larger than the contact time observed at $E_{\text {c.m. }}=200 \mathrm{MeV}$. The contact times observed in our TDHF calculations are of similar magnitude as those obtained by Simenel (see Fig. 38 of Ref. [33] and Fig. 8 of Ref. [91]).

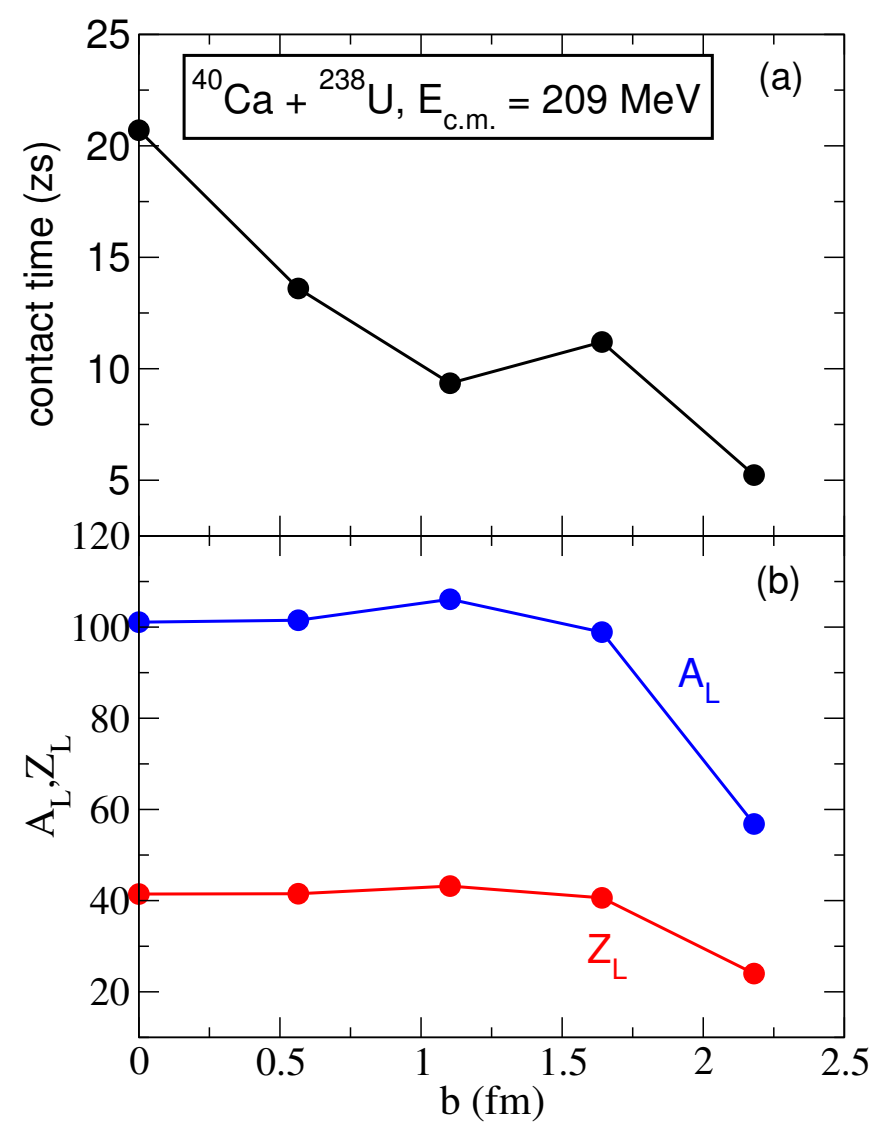

Figure 5: (a) contact time and (b) mass and charge of the light fragment as a function of impact parameter [72]

In Fig. 4p we show the corresponding masses $A_{\mathrm{L}}$ and charges $Z_{\mathrm{L}}$ of the light fragment. A comparison with Fig. 4 a reveals that mass and charge transfer are proportional to the contact time. For example, at $E_{\text {c.m. }}=200 \mathrm{MeV}$ there is very little mass transfer $\left(A_{\mathrm{L}}=41.4\right)$ and some charge pickup $\left(Z_{\mathrm{L}}=18.4\right)$. At $E_{\text {c.m. }}=208 \mathrm{MeV}$, the dramatic increase in contact time results in 
a large amount of both mass and charge transfer, $A_{\mathrm{L}}=98.7$ and $Z_{\mathrm{L}}=40.5$. At $E_{\text {c.m. }}=220 \mathrm{MeV}$ we find a light fragment mass $A_{\mathrm{L}}=107.2$ and charge $Z_{\mathrm{L}}=43.4$. Based on these results, we conclude that the energy region $E_{\text {c.m. }}=200-207 \mathrm{MeV}$ is likely dominated by deep-inelastic reactions while the energy region $E_{\mathrm{c} . \mathrm{m} .}=208-220 \mathrm{MeV}$ is dominated by quasifission. The TDHF calculations carried out at higher energies were fallowed up to $35 \mathrm{zs}$, in the energy range $E_{\text {c.m. }}=223-225 \mathrm{MeV}$ we observe one fragment in the exit channel; this is the fusion region. Naturally, quasifission is still possible at higher energies, but only for a certain range of nonzero impact parameters. Recently, we have developed an extension to TDHF theory via the use

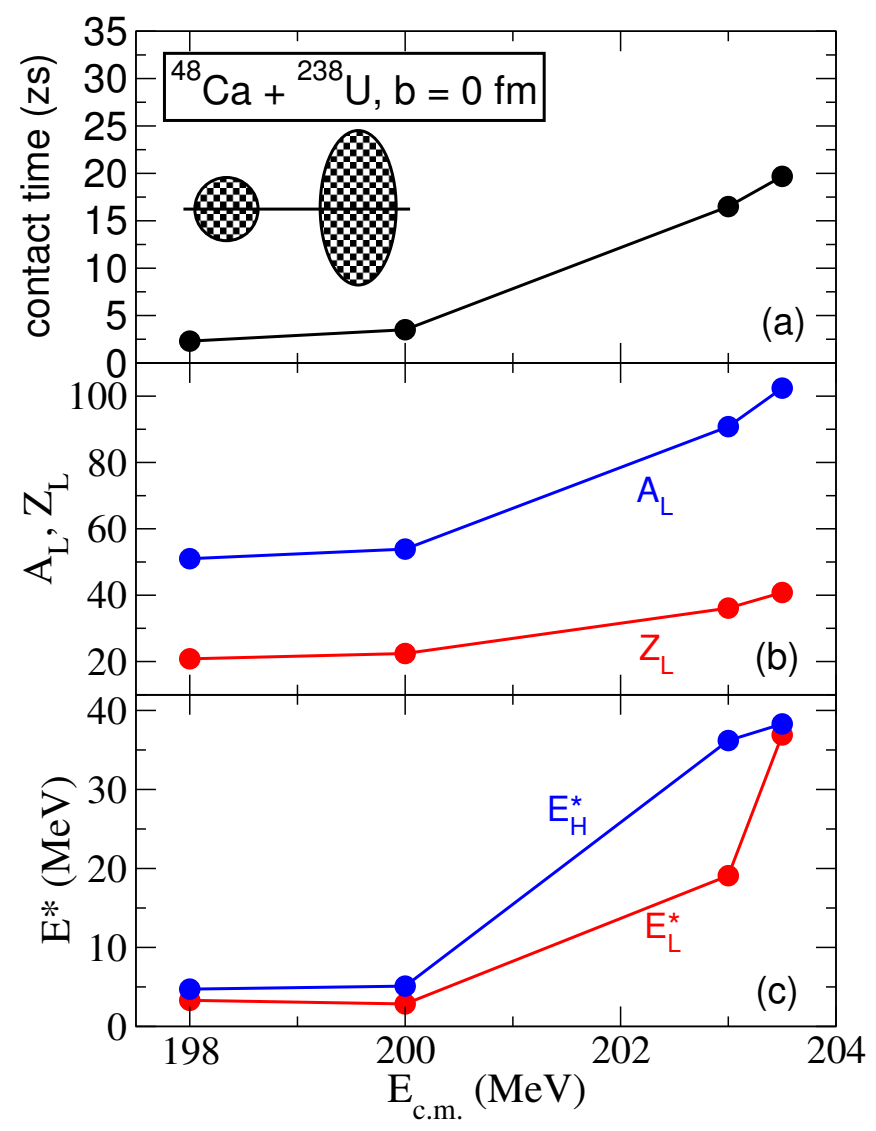

Figure 6: Several observables as a function of center-of-mass energy for a central collision of ${ }^{48} \mathrm{Ca}+{ }^{238} \mathrm{U}$ [72]. (a) contact time, (b) mass and charge of the light fragment, and (c) excitation energy of the light and heavy fragments. Quasifission dominates in a very narrow energy window, $E_{\mathrm{c} . \mathrm{m} .}=202-203.5 \mathrm{MeV}$.

of a density constraint to calculate fragment excitation energies directly from the TDHF time evolution [65]. In Fig. 4 we show the excitation energies of the light and heavy fragments as a function of the center-of-mass energy. For ${ }^{40} \mathrm{Ca}+{ }^{238} \mathrm{U}$, we find excitation energies in the QF region to be as high as $60 \mathrm{MeV}$ for the heavy fragment and $40 \mathrm{MeV}$ for the light fragment.

Figure 5 a shows the impact parameter dependence of the contact time, and Fig. 5p exhibits the corresponding masses $A_{\mathrm{L}}$ and charges $Z_{\mathrm{L}}$ of the light fragment for ${ }^{40} \mathrm{Ca}+{ }^{238} \mathrm{U}$ at a fixed center-of-mass energy $E_{\mathrm{c} . \mathrm{m} .}=209 \mathrm{MeV}$. We observe that the contact time decreases from its 
maximum around $21 \mathrm{zs}$ for the central collision to about $5 \mathrm{zs}$ for the impact parameter of $2.2 \mathrm{fm}$, where the last $\mathrm{QF}$ events are observed. The light fragment mass and charge stay flat around $A_{\mathrm{L}}=101.5-107.1$ and $Z_{\mathrm{L}}=40.6-42.2$ until a sudden drop for the largest impact parameter. Similar range of mass and charge combinations are also seen in Fig. 4 for a large energy range. The root of this behavior may be due to the fact that the $\mathrm{Zr}$ isotopes in the mass range 100-112 are strongly bound with a large prolate deformation around $\beta_{2}=0.42[92-95]$. Due to shell effects, these configurations may be energetically favorable during the QF dynamics. However, since TDHF theory does not include fluctuations only the most probable outcome is observed, while experimentally a distribution of masses will be measured.

We have repeated the same set of TDHF calculations for the neutron-rich system ${ }^{48} \mathrm{Ca}+{ }^{238} \mathrm{U}$, with the purpose of comparing the two systems. In Figures 6a and 6 6 we display the contact time and the light fragment mass / charge as a function of the center-of-mass energy for central collisions. These results are dramatically different as compared to the ${ }^{40} \mathrm{Ca}+{ }^{238} \mathrm{U}$ system: the quasifission region, as evidenced by long contact time and large mass transfer, is confined to a very narrow center-of-mass energy window, $E_{\mathrm{c} . \mathrm{m} .}=202-203.5 \mathrm{MeV}$. In this QF energy region, the contact time varies between (12.1-19.7) zs. Similarly, we find a light fragment mass range of $A_{\mathrm{L}}=78.7-102.4$ and charge range of $Z_{\mathrm{L}}=32.1-40.8$. Using our microscopic approach we have also calculated the excitation energy of the emerging fragments for the neutron-rich system ${ }^{48} \mathrm{Ca}+{ }^{238} \mathrm{U}$ as shown in Fig. 66. We find excitation energies in the QF region up to $40 \mathrm{MeV}$ for the heavy fragment as compared to $60 \mathrm{MeV}$ in the ${ }^{40} \mathrm{Ca}+{ }^{238} \mathrm{U}$ reaction.

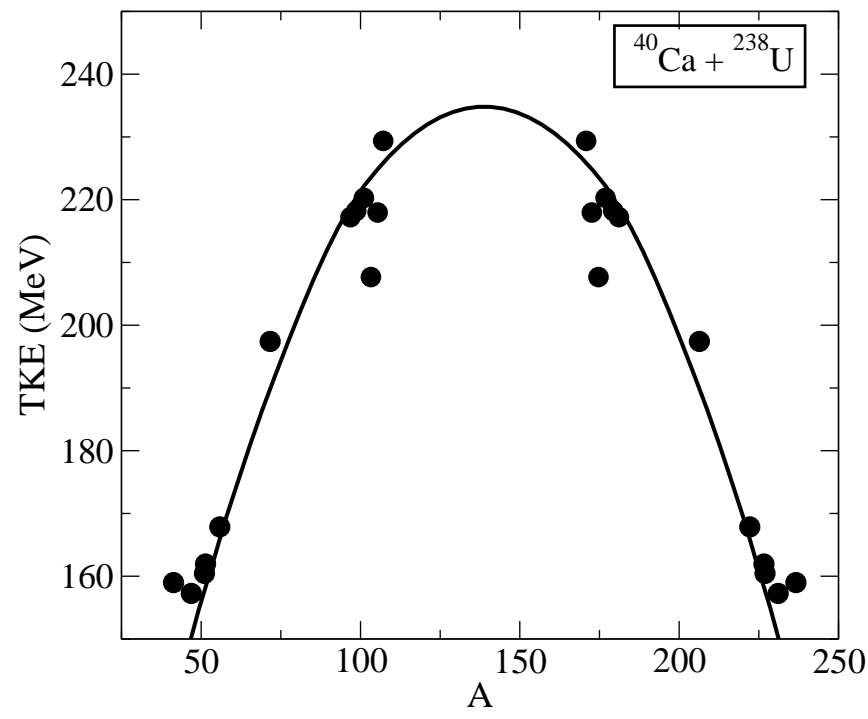

Figure 7: TKE of both the light and heavy fragments formed in ${ }^{40} \mathrm{Ca}+{ }^{238} \mathrm{U}$ central collisions. The filled circles represent results from TDHF calculations, and the solid line represents TKE values based on the Viola formula [96].

The contact times discussed above are long enough to enable the conversion of the initial relative kinetic energy into internal excitations. The total kinetic energy (TKE) distribution of the reaction products is one of the indicators of the source of the observed fragments. For quasifission, the TKE distribution is expected to be described by the Viola systematics [23, 97]. This indicates the TKE's of final fragments are primarily due to their Coulomb repulsion and do not 
carry a substantial portion of the initial relative kinetic energy. Experimentally, the measured total kinetic energy of the quasifission fragments in ${ }^{40,48} \mathrm{Ca}+{ }^{238} \mathrm{U}$ reactions is in relatively good agreement with the Viola systematics. The TDHF approach contains one-body dissipation mechanisms which are dominant at near-barrier energies and can be used to predict the final TKE of the fragments. The TKE of the fragments formed in ${ }^{40} \mathrm{Ca}+{ }^{238} \mathrm{U}$ have been computed for a range of central collisions up to $10 \%$ above the barrier. Figure 7 shows that the TDHF predictions of TKE are in excellent agreement with the Viola systematics. This is a further confirmation that the TDHF dynamics is providing a plausible description of the quasifission process.

An important observation of the above results is that the neutron-rich ${ }^{48} \mathrm{Ca}+{ }^{238} \mathrm{U}$ system shows considerably less QF in comparison to the stable ${ }^{40} \mathrm{Ca}+{ }^{238} \mathrm{U}$ system. Similarly, the excitation energies of the emerging QF fragments have considerably less intrinsic excitation. These results point to the conclusion that the neutron-rich system has a higher probability for leading to the formation of a superheavy element, as it was discovered experimentally. An alternative interpretation for the difference of $\mathrm{QF}$ in both reactions can be the magicity, which reduces dissipation and disappears in ${ }^{40} \mathrm{Ca}+{ }^{238} \mathrm{U}$ due to $\mathrm{N} / \mathrm{Z}$ equilibration [98].

\section{2. $C r+W$ systems}

Here we study the QF properties for neutron-poor and neutron-rich systems, ${ }^{50} \mathrm{Cr}+{ }^{180} \mathrm{~W}$ and ${ }^{54} \mathrm{Cr}+{ }^{186} \mathrm{~W}$, respectively. Both calculations were done at an energy $E_{\text {c.m. }} / V_{B}=1.13 \mathrm{MeV}$ corresponding to recent experiments [99], where $V_{B}$ denotes the Bass barrier.

Figure 8 shows the mass and charge transfer to the light $\mathrm{QF}$ fragment as a function of the contact time (left) and the rotation angle of the light fragment during the entire collision also as a function of contact time (right). We observe that the mass and charge of the light QF fragment increase with increased contact time, which corresponds to decrasing impact parameter. While not directly observable, calculations also show that the rotation angle increases almost linearly with increasing contact time corresponding to smaller impact parameters.
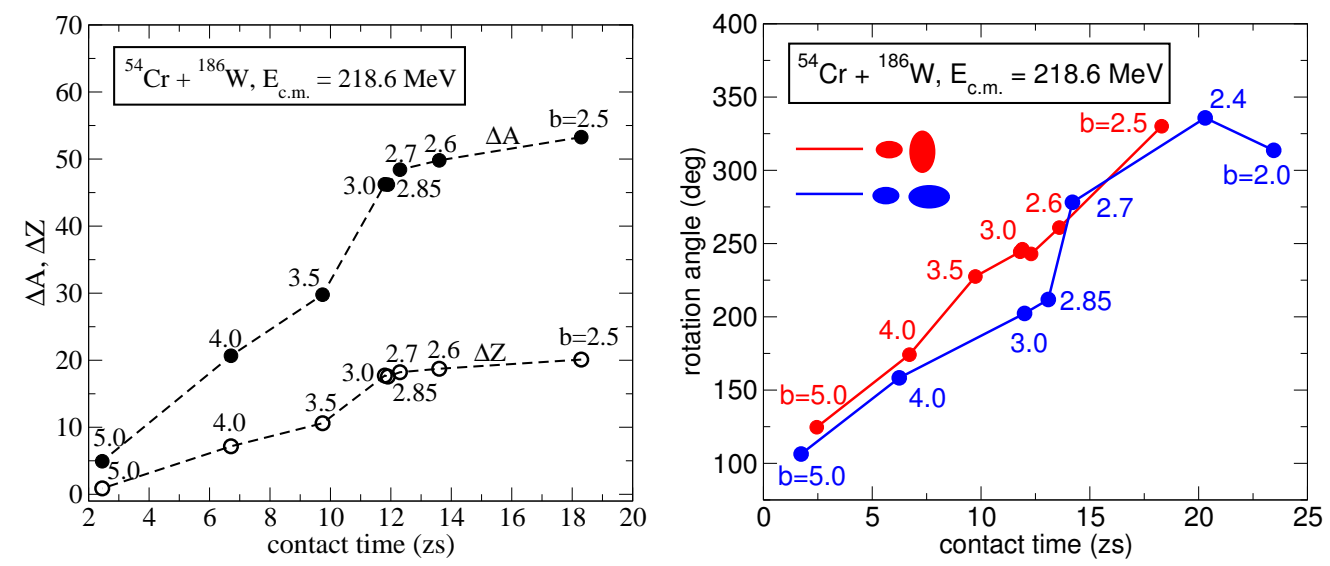

Figure 8: Left: Mass and charge differences, $\Delta A=A_{P L F}-54$ and $\Delta Z=Z_{P L F}-24$, for the tip-side collision of ${ }^{54} \mathrm{Cr}+{ }^{186} \mathrm{~W}$ at $E_{\mathrm{c} . \mathrm{m} .}=218.6 \mathrm{MeV}$. Right: TDHF calculations of the rotation angle of the final fragments as a function of contact time for ${ }^{54} \mathrm{Cr}+{ }^{186} \mathrm{~W}$ at $E_{\mathrm{c} . \mathrm{m} .}=218.6 \mathrm{MeV}$. The numbers associated with each point denote the corresponding impact parameter in $\mathrm{fm}$.

The rotation angle determines the scattering angles of the final QF fragments, which are observed experimentally. Measured mass-angle distributions (MADs) show the yield of mass- 
ratio, $M_{R}=m_{1} /\left(m_{1}+m_{2}\right)$, as a function of the c.m. angle of the quasifission products with masses $m_{1}$ and $m_{2}$. In the left plot of Fig. 9 we show the TDHF calculations of quasifission MADs for the reaction ${ }^{54} \mathrm{Cr}+{ }^{186} \mathrm{~W}$ at $E_{\mathrm{c} . \mathrm{m} .}=218.6 \mathrm{MeV}$, corresponding to the two orientations of the ${ }^{186} \mathrm{~W}$ nucleus. In the plot shown in the right side of Fig. 9 corresponding experimental MADs are shown [99]. The regions of MAD's near $M_{R}=0.2$ and $M_{R}=0.8$ correspond to elastic and quasielastic reactions, followed by transition to deep-inelastic reactions and subsequently quasifission. The transition from deep-inelastic to quasifission is correctly reproduced by TDHF as well as the general behavior of the MADs. Due to the fact that TDHF is a deterministic theory it will only give us the most probable outcome or path for the MADs rather than a full distribution. Similar MADs have been obtained for the ${ }^{40} \mathrm{Ca}+{ }^{238} \mathrm{U}$ system in Ref. [71].
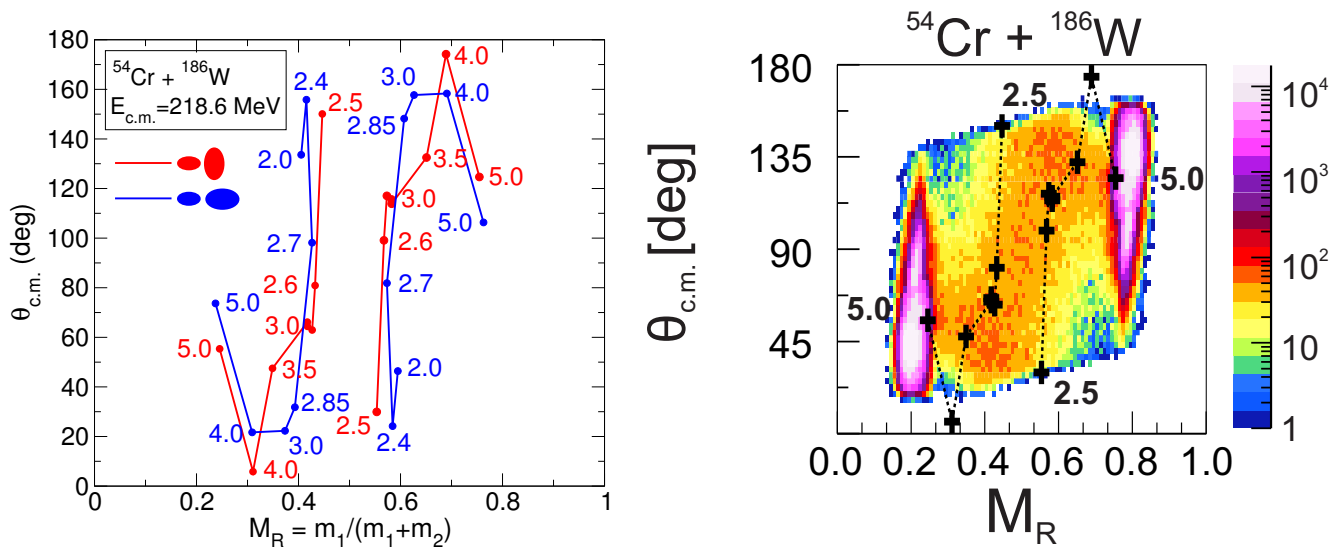

Figure 9: Left: TDHF calculation of MAD for the reaction products of the reaction ${ }^{54} \mathrm{Cr}+{ }^{186} \mathrm{~W}$ at $E_{\mathrm{c} . \mathrm{m} .}=218.6 \mathrm{MeV}$. Right: Experimental MADs [99] corresponding to the same reaction. The black crosses show the TDHF results for the tip-side collision with the corresponding impact parameter values in fm's.

\section{Ingredients for evaluating $\boldsymbol{P}_{\mathrm{CN}}$}

In this section we will discuss the possibility of calculating some of the ingredients that go into the computation of $P_{\mathrm{CN}}$ which is the probability that the system evolves into a fused system rather than quasifission. The two main references used in the discussion of $P_{\mathrm{CN}}$ for quasifission are [86, 100].

\subsection{Moment of inertia}

During the collision process the nuclear densities, as described by TDHF time-evolution, undergo complicated shape changes, rotations, etc. finally leading to two separated final fragments identified as quasifission due to the long contact-time for the reaction as well as the mass/charge of the fragments.

We have realized that the proper way to calculate the moment-of-inertia for such timedependent densities is to directly diagonalize the moment-of-inertia tensor

$$
\mathfrak{J}_{i j} / m=\int d^{3} r \rho_{T D H F}(\mathbf{r}, t)\left(r^{2} \delta_{i j}-x_{i} x_{j}\right),
$$


where $\rho_{T D H F}$ is the number-density in units of $\left(\mathrm{N} / \mathrm{fm}^{3}\right), \mathrm{m}$ is the nucleon mass, and $x_{i}$ denote the Cartesian coordinates. The TDHF calculations are done in three-dimensional Cartesian geometry [59]. Numerical diagonalization of this $3 \times 3$ matrix gives the 3 eigenvalues, one corresponding the the moment-of-inertia for the nuclear system rotating about the symmetry axis, and the other two for rotations about axes perpendicular to the symmetry axis. We denote these by $\mathfrak{I}_{\|}$and $\mathfrak{I}_{\perp}$. Naturally, for triaxial density distributions the two perpendicular components are not exactly equal but for practical calculations they are close enough and always larger than the parallel component.

Using the time-dependent moment-of-inertial obtained from the TDHF collision one can calculate the so-called effective moment-of-inertia

$$
\frac{1}{\mathfrak{J}_{\text {eff }}}=\frac{1}{\mathfrak{J}_{\|}}-\frac{1}{\mathfrak{J}_{\perp}} .
$$

In literature [86, 100] what is usually given is the ratio $\mathfrak{J}_{0} / \mathfrak{J}_{\text {eff }}$ at the saddle point of the fission barrier, where $\mathfrak{J}_{0}$ is the moment-of-inertia of spherical nucleus with the same mass. This ratio is to be constant for impact parameters leading to quasifission $\left(J>J_{C N}\right)$, where $J_{C N}$ is the largest $J$ value resulting in compound nucleus formation. The expression for the moment-of-inertia for a rigid sphere is given by $\mathfrak{J}_{0} / m=2 / 5 A R_{0}^{2}$, which in units of $\hbar^{2} \mathrm{MeV}^{-1}$ can also be written as

$$
\mathfrak{J}_{0}=\hbar^{2}\left(2 / 5 A R_{0}^{2}\right) /\left(\hbar^{2} / m\right)
$$

In Ref. [100] the $R_{0}$ was chosen to be $R_{0}=1.225 A^{1 / 3}$. With the choice of $\hbar^{2} / \mathrm{m}=$ $41.471 \mathrm{MeV} \cdot \mathrm{fm}^{2}$, corresponding to the value used in the Skyrme SLy4d interaction, for $A=286$ we get $\mathfrak{I}_{0}=179.693 \hbar^{2} \cdot \mathrm{MeV}^{-1}$

In Fig. 10 we show the time-evolution of the ratio $\mathfrak{J}_{0} / \mathfrak{J}_{\text {eff }}$ for the TDHF collision of the ${ }^{48} \mathrm{Ca}+{ }^{238} \mathrm{U}$ system for central collision at an energy $E_{\mathrm{c} . \mathrm{m} .}=203 \mathrm{MeV}$. We see that during the times that possibly correspond to the vicinity of the saddle point the ratio appears to be smaller than the traditionally used value of 1.5 . We will come back to the discussion of finding the saddle point later in this document. Before we end this section we should also point out that we having the numerical values for $\mathfrak{J}_{\perp}$ also allows the computation of the rotational energy

$$
E_{\text {rot }}=\frac{\ell(\ell+1) \hbar^{2}}{2 \mathfrak{J}_{\perp}} .
$$

\subsection{Quasifission barrier}

Recently, the newly developed density-constrained TDHF method has proven to be a powerful method of obtaining fusion barriers microscopically from TDHF time-evolution of the nuclear densities. This is a parameter free way of obtaining these barriers.

In principle, the same approach can be used to compute the underlying barrier during the quasifission dynamics. From this we may be able to get most of the other ingredients of computing

$$
K_{0, \ell}=T \mathfrak{J}_{\text {eff }} / \hbar^{2},
$$

where $T$ is the nuclear temperature involving various quantities such as $E^{*}$, barrier height, and others. In practice the $\ell$ dependence of this expression is ignored, which may be a reasonable approximation. The computation of the barrier will be very time-consuming but it may give us a better understanding of the quasifission process. 


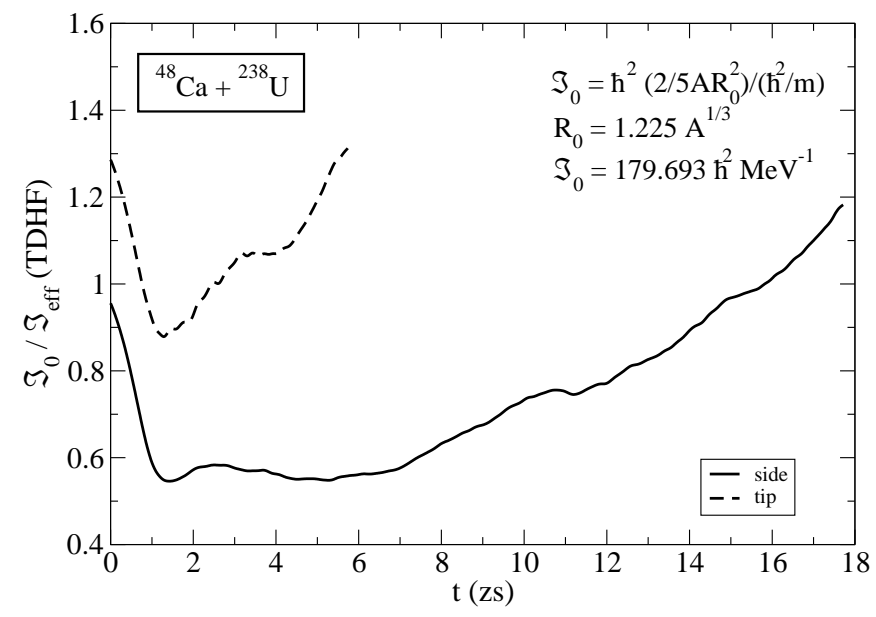

Figure 10: TDHF results showing the time-dependence of the ratio $\mathfrak{J}_{0} / \mathfrak{J}_{\text {eff }}$ for the ${ }^{48} \mathrm{Ca}+{ }^{238} \mathrm{U}$ system at energy $E_{\text {c.m. }}=203 \mathrm{MeV}$ and zero impact parameter.

\section{Conclusions}

Recent TDHF calculations of phenomena related to SHE searches show that TDHF can be a valuable tool for elucidating some of the underlying physics problems encountered. As a microscopic theory with no free parameters, where the effective nucleon-nucleon interaction is only fitted to the static properties of a few nuclei, these results are very promising.

\section{Acknowledgments}

We gratefully acknowledge discussions with C. Simenel, Z. Kohley, and W. Loveland. This work has been supported by the U.S. Department of Energy under Grant No. DE-FG0296ER40975 with Vanderbilt University.

\section{References}

[1] P. Armbruster, Ann. Rev. Nucl. Part. Sci. 35 (1985) 135. doi 10.1146/annurev.ns.35.120185.001031

[2] S. Hofmann, Rep. Prog. Phys. 61 (1998) 639. doi 10.1088/0034-4885/61/6/002

[3] S. Hofmann, G. Münzenberg, Rev. Mod. Phys. 72 (2000) 733. doi 10.1103/RevModPhys.72.733

[4] Y. Oganessian, J. Phys. G 34 (2007) R165. doi 10.1088/0954-3899/34/4/R01

[5] Yu. Ts. Oganessian, F. Sh. Abdullin, P. D. Bailey, D. E. Benker, M. E. Bennett, S. N. Dmitriev, J. G. Ezold, J. H. Hamilton, R. A. Henderson, M. G. Itkis, Y. V. Lobanov, A. N. Mezentsev, K. J. Moody, S. L. Nelson, A. N. Polyakov, C. E. Porter, A. V. Ramayya, F. D. Riley, J. B. Roberto, M. A. Ryabinin, K. P. Rykaczewski, R. N. Sagaidak, D. A. Shaughnessy, I. V. Shirokovsky, M. A. Stoyer, V. G. Subbotin, R. Sudowe, A. M. Sukhov, Y. S. Tsyganov, V. K. Utyonkov, A. A. Voinov, G. K. Vostokin, P. A. Wilk, Phys. Rev. Lett. 104 (2010) 142502. doi 10.1103/PhysRevLett.104.142502

[6] Y. T. Oganessian, F. S. Abdullin, C. Alexander, J. Binder, R. A. Boll, S. N. Dmitriev, J. Ezold, K. Felker, J. M. Gostic, R. K. Grzywacz, J. H. Hamilton, R. A. Henderson, M. G. Itkis, K. Miernik, D. Miller, K. J. Moody, A. N. Polyakov, A. V. Ramayya, J. B. Roberto, M. A. Ryabinin, K. P. Rykaczewski, R. N. Sagaidak, D. A. Shaughnessy, I. V. Shirokovsky, M. V. Shumeiko, M. A. Stoyer, N. J. Stoyer, V. G. Subbotin, A. M. Sukhov, Y. S. Tsyganov, V. K. Utyonkov, A. A. Voinov, G. K. Vostokin, Phys. Rev. Lett. 109 (2012) 162501 doi 10.1103/PhysRevLett.109.162501 
[7] J. Khuyagbaatar, A. Yakushev, C. E. Düllmann, D. Ackermann, L.-L. Andersson, M. Asai, M. Block, R. A. Boll, H. Brand, D. M. Cox, M. Dasgupta, X. Derkx, A. Di Nitto, K. Eberhardt, J. Even, M. Evers, C. Fahlander, U. Forsberg, J. M. Gates, N. Gharibyan, P. Golubev, K. E. Gregorich, J. H. Hamilton, W. Hartmann, R.-D. Herzberg, F. P. Heßberger, D. J. Hinde, J. Hoffmann, R. Hollinger, A. Hübner, E. Jäger, B. Kindler, J. V. Kratz, J. Krier, N. Kurz, M. Laatiaoui, S. Lahiri, R. Lang, B. Lommel, M. Maiti, K. Miernik, S. Minami, A. Mistry, C. Mokry, H. Nitsche, J. P. Omtvedt, G. K. Pang, P. Papadakis, D. Renisch, J. Roberto, D. Rudolph, J. Runke, K. P. Rykaczewski, L. G. Sarmiento, M. Schädel, B. Schausten, A. Semchenkov, D. A. Shaughnessy, P. Steinegger, J. Steiner, E. E. Tereshatov, P. Thörle-Pospiech, K. Tinschert, T. Torres De Heidenreich, N. Trautmann, A. Türler, J. Uusitalo, D. E. Ward, M. Wegrzecki, N. Wiehl, S. M. Van Cleve, V. Yakusheva, Phys. Rev. Lett. 112 (2014) 172501. doi 10.1103/PhysRevLett.112.172501

[8] A. Staszczak, A. Baran, W. Nazarewicz, Phys. Rev. C 87 (2013) 024320. doi 10.1103/PhysRevC.87.024320

[9] S. Ćwiok, P.-H. Heenen, W. Nazarewicz, Nature 433 (2005) 705. doi 10.1038/nature03336

[10] T. Bürvenich, M. Bender, J. A. Maruhn, P.-G. Reinhard, Phys. Rev. C 69 (2004) 014307. doi 10.1103/PhysRevC.69.014307

[11] Peter Möller, Arnold J. Sierk, Takatoshi Ichikawa, Akira Iwamoto, Ragnar Bengtsson, Henrik Uhrenholt, Sven Aberg, Phys. Rev. C 79 (2009) 064304. doi 10.1103/PhysRevC.79.064304

[12] J. C. Pei, W. Nazarewicz, J. A. Sheikh, A. K. Kerman, Phys. Rev. Lett. 102 (2009) 192501. doi 10.1103/PhysRevLett.102.192501

[13] H. Abusara, A. V. Afanasjev, P. Ring, Phys. Rev. C 85 (2012) 024314. doi 10.1103/PhysRevC.85.024314

[14] S. Hofmann, F. P. Heßberger, D. Ackermann, G. Münzenberg, S. Antalic, P. Cagarda, B. Kindler, J. Kojouharova, M. Leino, B. Lommel, R. Mann, A. G. Popeko, S. Reshitko, S. Śaro, J. Uusitalo, A. V. Yeremin, Eur. Phys. J. A 14 (2002) 147. doi 10.1140/epja/i2001-10119-x

[15] R. S. Naik, W. Loveland, P. H. Sprunger, A. M. Vinodkumar, D. Peterson, C. L. Jiang, S. Zhu, X. Tang, E. F. Moore, P. Chowdhury, Phys. Rev. C 76 (2007) 054604. doi 10.1103/PhysRevC.76.054604

[16] Y. T. Oganessian, V. K. Utyonkov, Y. V. Lobanov, F. S. Abdullin, A. N. Polyakov, I. Shirokovsky, Y. S. Tsyganov, G. G. Gulbekian, S. L. Bogomolov, B. N. Gikal, A. N. Mezentsev, S. Iliev, V. G. Subbotin, A. M. Sukhov, A. A. Voinov, G. V. Buklanov, K. Subotic, V. I. Zagrebaev, M. G. Itkis, J. B. Patin, K. J. Moody, J. F. Wild, M. A. Stoyer, N. J. Stoyer, D. A. Shaughnessy, J. M. Kenneally, P. A. Wilk, R. W. Lougheed, R. I. Il'kaev, S. P. Vesnovskii, Phys. Rev. C 70 (2004) 064609. doi 10.1103/PhysRevC.70.064609

[17] S. Hofmann, D. Ackermann, S. Antalic, H. G. Burkhard, V. F. Comas, R. Dressler, Z. Gan, S. Heinz, J. A. Heredia, F. P. Heßberger, J. Khuyagbaatar, B. Kindler, I. Kojouharov, P. Kuusiniemi, M. Leino, B. Lommel, R. Mann, G. Münzenberg, K. Nishio, A. G. Popeko, S. Saro, H. J. Schött, B. Streicher, B. Sulignano, J. Uusitalo, M. Venhart, A. V. Yeremin, Eur. Phys. J. A 32 (2007) 251. doi 10.1140/epja/i2007-10373-X

[18] D. J. Hinde, M. Dasgupta, J. R. Leigh, J. P. Lestone, J. C. Mein, C. R. Morton, J. O. Newton, H. Timmers, Phys. Rev. Lett. 74 (1995) 1295. doi 10.1103/PhysRevLett.74.1295

[19] D. J. Hinde, M. Dasgupta, A. Mukherjee, $\quad$ Phys. Rev. Lett. 89 (2002) 282701. doi 10.1103/PhysRevLett.89.282701

[20] I. M. Itkis, E. M. Kozulin, M. G. Itkis, G. N. Knyazheva, A. A. Bogachev, E. V. Chernysheva, L. Krupa, Y. T. Oganessian, V. I. Zagrebaev, A. Y. Rusanov, F. Goennenwein, O. Dorvaux, L. Stuttgé, F. Hanappe, E. Vardaci, E. Goés de Brennand, Phys. Rev. C 83 (2011) 064613. doi 10.1103/PhysRevC.83.064613

[21] G. N. Knyazheva, E. M. Kozulin, R. N. Sagaidak, A. Y. Chizhov, M. G. Itkis, N. A. Kondratiev, V. M. Voskressensky, A. M. Stefanini, B. R. Behera, L. Corradi, E. Fioretto, A. Gadea, A. Latina, S. Szilner, M. Trotta, S. Beghini, G. Montagnoli, F. Scarlassara, F. Haas, N. Rowley, P. R. S. Gomes, A. Szanto de Toledo, Phys. Rev. C 75 (2007) 064602. doi 10.1103/PhysRevC.75.064602

[22] W. Q. Shen, J. Albinski, A. Gobbi, S. Gralla, K. D. Hildenbrand, N. Herrmann, J. Kuzminski, W. F. J. Müller, H. Stelzer, J. Töke, B. B. Back, S. Bjørnholm, S. P. Sørensen, Phys. Rev. C 36 (1987) 115. doi 10.1103/PhysRevC.36.115

[23] K. Nishio, S. Mitsuoka, I. Nishinaka, H. Makii, Y. Wakabayashi, H. Ikezoe, K. Hirose, T. Ohtsuki, Y. Aritomo, S. Hofmann, Phys. Rev. C 86 (2012) 034608. doi 10.1103/PhysRevC.86.034608

[24] E. Williams, D. J. Hinde, M. Dasgupta, R. du Rietz, I. P. Carter, M. Evers, D. H. Luong, S. D. McNeil, D. C. Rafferty, K. Ramachandran, A. Wakhle, Phys. Rev. C 88 (2013) 034611. doi 10.1103/PhysRevC.88.034611

[25] G. G. Adamian, N. V. Antonenko, W. Scheid, Phys. Rev. C 68 (2003) 034601. doi 10.1103/PhysRevC.68.034601

[26] G. G. Adamian, N. V. Antonenko, W. Scheid, Eur. Phys. J. A 41 (2009) 235. doi 10.1140/epja/i2009-10795-4

[27] A. K. Nasirov, G. Giardina, G. Mandaglio, M. Manganaro, F. Hanappe, S. Heinz, S. Hofmann, A. I. Muminov, W. Scheid, Phys. Rev. C 79 (2009) 024606. doi 10.1103/PhysRevC.79.024606

[28] Valery Zagrebaev, Walter Greiner, J. Phys. G 34 (2007) 2265.

[29] G. Fazio, G. Giardina, G. Mandaglio, R. Ruggeri, A. I. Muminov, A. K. Nasirov, Y. T. Oganessian, A. G. Popeko, R. N. Sagaidak, A. V. Yeremin, S. Hofmann, F. Hanappe, C. Stodel, Phys. Rev. C 72 (2005) 064614. doi $10.1103 /$ PhysRevC.72.064614 
[30] Y. Aritomo, Phys. Rev. C 80 (2009) 064604. doi 10.1103/PhysRevC.80.064604

[31] V. I. Zagrebaev, W. Greiner, Phys. Rev. C 87 (2013) 034608. doi $10.1103 /$ PhysRevC.87.034608

[32] J. W. Negele, Rev. Mod. Phys. 54 (1982) 913. doi 10.1103/RevModPhys.54.913

[33] C. Simenel, Eur. Phys. J. A 48 (2012) 152. doi 10.1140/epja/i2012-12152-0

[34] A. S. Umar, V. E. Oberacker, Phys. Rev. C 71 (2005) 034314. doi 10.1103/PhysRevC.71.034314

[35] J. A. Maruhn, P. G. Reinhard, P. D. Stevenson, J. Rikovska Stone, M. R. Strayer, Phys. Rev. C 71 (2005) 064328. doi 10.1103/PhysRevC.71.064328

[36] T. Nakatsukasa, K. Yabana, Phys. Rev. C 71 (2005) 024301. doi 10.1103/PhysRevC.71.024301

[37] C. Simenel, P. Chomaz, Phys. Rev. C 68 (2003) 024302. doi 10.1103/PhysRevC.68.024302

[38] P.-G. Reinhard, P. D. Stevenson, D. Almehed, J. A. Maruhn, M. R. Strayer, Phys. Rev. E 73 (2006) 036709. doi 10.1103/PhysRevE.73.036709

[39] P.-G. Reinhard, Lu Guo, J. Maruhn, Eur. Phys. J. A 32 (2007) 19. doi 10.1140/epja/i2007-10366-9

[40] E. B. Suckling, P. D. Stevenson, EPL 90 (2010) 12001. doi 10.1209/0295-5075/90/12001

[41] J. C. Pei, F. R. Xu, P. D. Stevenson, Phys. Rev. C 71 (2005) 034302. doi 10.1103/PhysRevC.71.034302

[42] F. R. Xu, J. C. Pei, P. D. Stevenson, J. Phys. G 31 (2005) S1541. doi 10.1088/0954-3899/31/10/028

[43] A. S. Umar, V. E. Oberacker, J. A. Maruhn, P.-G. Reinhard, Phys. Rev. C 81 (2010) 064607. doi 10.1103/PhysRevC.81.064607

[44] Kouhei Washiyama, Denis Lacroix, Phys. Rev. C 78 (2008) 024610. doi 10.1103/PhysRevC.78.024610

[45] R. Keser, A. S. Umar, V. E. Oberacker, Phys. Rev. C 85 (2012) 044606. doi 10.1103/PhysRevC.85.044606

[46] Cédric Simenel, Benoit Avez, Intl. J. Mod. Phys. E 17 (2008) 31. doi 10.1142/S0218301308009525

[47] C. Simenel, M. Dasgupta, D. J. Hinde, E. Williams, Phys. Rev. C 88 (2013) 064604. doi $10.1103 /$ PhysRevC.88.064604

[48] A. S. Umar, C. Simenel, V. E. Oberacker, Phys. Rev. C 89 (2014) 034611. doi 10.1103/PhysRevC.89.034611

[49] C. Simenel, Phys. Rev. Lett. 105 (2010) 192701. doi 10.1103/PhysRevLett.105.192701

[50] C. Simenel, Phys. Rev. Lett. 106 (2011) 112502. doi 10.1103/PhysRevLett.106.112502

[51] A. S. Umar, V. E. Oberacker, J. A. Maruhn, Eur. Phys. J. A 37 (2008) 245. doi 10.1140/epja/i2008-10614-6

[52] Kazuyuki Sekizawa, Kazuhiro Yabana, Phys. Rev. C 88 (2013) 014614. doi $10.1103 /$ PhysRevC.88.014614

[53] G. Scamps, D. Lacroix, Phys. Rev. C 87 (2013) 014605. doi 10.1103/PhysRevC.87.014605

[54] C. Simenel, A. S. Umar, Phys. Rev. C 89 (2014) 031601. doi 10.1103/PhysRevC.89.031601

[55] M. Tohyama, A. S. Umar, Phys. Lett. B 549 (2002) 72. doi $10.1016 /$ S0370-2693(02)02885-X

[56] M. Tohyama, A. S. Umar, Phys. Lett. B 516 (2001) 415. doi 10.1016/S0370-2693(01)00925-X

[57] D. Lacroix, S. Ayik, Eur. Phys. J. A 50 (2014) 95. doi 10.1140/epja/i2014-14095-8

[58] A. S. Umar, M. R. Strayer, J. S. Wu, D. J. Dean, M. C. Güçlü, Phys. Rev. C 44 (1991) 2512. doi 10.1103/PhysRevC.44.2512

[59] A. S. Umar, V. E. Oberacker, Phys. Rev. C 73 (2006) 054607. doi 10.1103/PhysRevC.73.054607

[60] J. A. Maruhn, P.-G. Reinhard, P. D. Stevenson, A. S. Umar, Comp. Phys. Comm. 185 (2014) 2195. doi $10.1016 /$ j.cpc.2014.04.008

[61] E. Chabanat, P. Bonche, P. Haensel, J. Meyer, R. Schaeffer, Nucl. Phys. A 635 (1998) 231. doi 10.1016/S03759474(98)00180-8

[62] P. Klüepfel, P.-G. Reinhard, T. J. Bürvenich, J. A. Maruhn, Phys. Rev. C 79 (2009) 034310. doi $10.1103 /$ PhysRevC.79.034310

[63] M. Kortelainen, T. Lesinski, J. More, W. Nazarewicz, J. Sarich, N. Schunck, M. V. Stoitsov, S. Wild, Phys. Rev. C 82 (2010) 024313. doi 10.1103/PhysRevC.82.024313

[64] A. S. Umar, V. E. Oberacker, Phys. Rev. C 74 (2006) 021601. doi 10.1103/PhysRevC.74.021601

[65] A. S. Umar, V. E. Oberacker, J. A. Maruhn, P.-G. Reinhard, Phys. Rev. C 80 (2009) 041601. doi 10.1103/PhysRevC.80.041601

[66] A. S. Umar, V. E. Oberacker, Phys. Rev. C 74 (2006) 024606. doi 10.1103/PhysRevC.74.024606

[67] A. S. Umar, V. E. Oberacker, Phys. Rev. C 77 (2008) 064605. doi 10.1103/PhysRevC.77.064605

[68] A. S. Umar, V. E. Oberacker, Eur. Phys. J. A 39 (2009) 243. doi 10.1140/epja/i2008-10712-5

[69] C. Simenel, R. Keser, A. S. Umar, V. E. Oberacker, Phys. Rev. C 88 (2013) 024617. doi 10.1103/PhysRevC.88.024617

[70] A. S. Umar, V. E. Oberacker, C. J. Horowitz, Phys. Rev. C 85 (2012) 055801. doi 10.1103/PhysRevC.85.055801

[71] A. Wakhle, C. Simenel, D. J. Hinde, M. Dasgupta, M. Evers, D. H. Luong, R. du Rietz, E. Williams, Phys. Rev. Lett. 113 (2014) 182502. doi 10.1103/PhysRevLett.113.182502

[72] V. E. Oberacker, A. S. Umar, C. Simenel, Phys. Rev. C 90 (2014) 054605. doi 10.1103/PhysRevC.90.054605

[73] C. Bottcher, M. R. Strayer, A. S. Umar, P.-G. Reinhard, Phys. Rev. A 40 (1989) 4182. doi 10.1103/PhysRevA.40.4182

[74] M. R. Strayer, C. Bottcher, V. E. Oberacker, A. S. Umar, Phys. Rev. A 41 (1990) 1399. doi 10.1103/PhysRevA.41.1399 
[75] R. Y. Cusson, P.-G. Reinhard, M. R. Strayer, J. A. Maruhn, W. Greiner, Z. Phys. A 320 (1985) 475 doi $10.1007 /$ BF01415725

[76] A. S. Umar, M. R. Strayer, R. Y. Cusson, P.-G. Reinhard, D. A. Bromley, Phys. Rev. C 32 (1985) 172. doi $10.1103 /$ PhysRevC.32.172

[77] X. Jiang, J. A. Maruhn, S. Yan, Microscopic study of noncentral effects in heavy-ion fusion reactions with spherical nuclei, Phys. Rev. C 90 (2014) 064618. doi 10.1103/PhysRevC.90.064618

[78] K. Hagino, Y. Watanabe, Phys. Rev. C 76 (2007) 021601. doi 10.1103/PhysRevC.76.021601

[79] A. S. Umar, M. R. Strayer, P.-G. Reinhard, Phys. Rev. Lett. 56 (1986) 2793. doi 10.1103/PhysRevLett.56.2793

[80] P.-G. Reinhard, A. S. Umar, K. T. R. Davies, M. R. Strayer, S. J. Lee, Phys. Rev. C 37 (1988) 1026 doi 10.1103/PhysRevC.37.1026

[81] A. S. Umar, M. R. Strayer, P.-G. Reinhard, K. T. R. Davies, S.-J. Lee, Phys. Rev. C 40 (1989) 706. doi 10.1103/PhysRevC.40.706

[82] S. Fracasso, E. B. Suckling, P. D. Stevenson, Phys. Rev. C 86 (2012) 044303. doi 10.1103/PhysRevC.86.044303

[83] M. Tohyama, A. S. Umar, Phys. Rev. C 65 (2002) 037601. doi 10.1103/PhysRevC.65.037601

[84] B. Avez, C. Simenel, P. Chomaz, Phys. Rev. C 78 (2008) 044318. doi 10.1103/PhysRevC.78.044318

[85] I. Stetcu, A. Bulgac, P. Magierski, K. J. Roche, Phys. Rev. C 84 (2011) 051309 doi 10.1103/PhysRevC.84.051309

[86] R. Yanez, W. Loveland, J. S. Barrett, L. Yao, B. B. Back, S. Zhu, T. L. Khoo, Phys. Rev. C 88 (2013) 014606 doi 10.1103/PhysRevC.88.014606

[87] M. G. Itkis, A. A. Bogatchev, I. M. Itkis, M. Jandel, J. Kliman, G. N. Kniajeva, N. A. Kondratiev, I. V. Korzyukov, E. M. Kozulin, L. Krupa, Y. T. Oganessian, I. V. Pokrovski, V. A. Ponomarenko, E. V. Prokhorova, A. Y. Rusanov, V. M. Voskresenski, A. A. Goverdovski, F. Hanappe, T. Materna, N. Rowley, L. Stuttge, G. Giardina, K. J. Moody, J. Nucl. Radiochem. 3 (2002) 57. doi 10.14494/jnrs2000.3.57

[88] M. G. Itkis, J. Äystö, S. Beghini, A. Bogachev, L. Corradi, O. Dorvaux, A. Gadea, G. Giardina, F. Hanappe, I. Itkis, M. Jandel, J. Kliman, S. Khlebnikov, G. Kniajeva, N. Kondratiev, E. Kozulin, L. Krupa, A. Latina, T. Materna, G. Montagnoli, Y. Oganessian, I. Pokrovsky, E. Prokhorova, N. Rowley, V. Rubchenya, A. Rusanov, R. Sagaidak, F. Scarlassara, A. Stefanini, L. Stuttge, S. Szilner, M. Trotta, W. Trzaska, D. Vakhtin, A. Vinodkumar, V. Voskressenski, V. Zagrebaev, Nucl. Phys. A 734 (2004) 136. doi 10.1016/j.nuclphysa.2004.01.022

[89] C. Simenel, P. Chomaz, G. de France, Phys. Rev. Lett. 93 (2004) 102701. doi 10.1103/PhysRevLett.93.102701

[90] Ka-Hae Kim, Takaharu Otsuka, Paul Bonche, J. Phys. G 23 (1997) 1267. doi 10.1088/0954-3899/23/10/014

[91] Cédric Simenel, Aditya Wakhle, B. Avez, D. J. Hinde, R. du Rietz, M. Dasgupta, M. Evers, C. J. Lin, D. H. Luong, Eur. Phys. J Web. Conf. 38 (2012) 09001. doi 10.1051/epjconf/20123809001

[92] P. Möller, J. R. Nix, W. D. Myers, W. J. Swiatecki, At. Data Nucl. Data Tables 59 (1995) 185 doi 10.1006/adnd.1995.1002

93] G. A. Lalazissis, S. Raman, P. Ring, At. Data Nucl. Data Tables 71 (1999) 1. doi 10.1006/adnd.1998.0795

[94] A. Blazkiewicz, V. E. Oberacker, A. S. Umar, M. Stoitsov, Phys. Rev. C 71 (2005) 054321. doi 10.1103/PhysRevC.71.054321

[95] J. K. Hwang, A. V. Ramayya, J. H. Hamilton, Y. X. Luo, A. V. Daniel, G. M. Ter-Akopian, J. D. Cole, S. J. Zhu, Phys. Rev. C 73 (2006) 044316. doi 10.1103/PhysRevC.73.044316

[96] V. E. Viola, K. Kwiatkowski, M. Walker, Phys. Rev. C 31 (1985) 1550. doi 10.1103/PhysRevC.31.1550

[97] J. Töke, R. Bock, G. Dai, A. Gobbi, S. Gralla, K. Hildenbrand, J. Kuzminski, W. Müller, A. Olmi, H. Stelzer, B. Back, S. Bjørnholm, Nucl. Phys. A 440 (1985) 327. doi 10.1016/0375-9474(85)90344-6

[98] C. Simenel, D. J. Hinde, R. du Rietz, M. Dasgupta, M. Evers, C. J. Lin, D. H. Luong, A. Wakhle, Phys. Lett. B 710 (2012) 607. doi 10.1016/j.physletb.2012.03.063

[99] K. Hammerton, Z. Kohley, D. J. Hinde, M. Dasgupta, A. Wakhle, E. Williams, V. E. Oberacker, A. S. Umar, I. P. Carter, K. J. Cook, J. Greene, D. Y. Jeong, D. H. Luong, S. D. McNeil, C. Palshetkar, D. C. Rafferty, C. Simenel, K. Stiefel, submitted (2014).

[100] M. Tsang, H. Utsunomiya, C. Gelbke, W. Lynch, B. Back, S. Saini, P. Baisden, M. McMahan, Phys. Lett. B 129 (1983) 18. doi $10.1016 / 0370-2693(83) 90719-0$ 